\title{
Propagation of a laser pulse in a three-level cascade atomic medium under conditions of electromagnetically induced transparency
}

\author{
Hoang Minh Dong ${ }^{1,2}$, Le Van Doai ${ }^{1}$, Vu Ngoc Sau ${ }^{1}$, Dinh Xuan Khoa ${ }^{1}$ and Nguyen Huy Bang ${ }^{*}, 1$ \\ ${ }^{1}$ Vinh University, 182 Le Duan Street, Vinh City, Vietnam. \\ ${ }^{2}$ Central Region Transport College, Vinh City, Vietnam
}

Received August 27, 2016; accepted September 29, 2016; published September 30, 2016

\begin{abstract}
We study the propagation dynamics of a single pair of probe and coupling laser pulses in a three-level cascade atomic medium by numerical solving the Maxwell-Bloch equations for atoms and fields. There are investigated influences of pulse duration, intensity and pulse area of a coupling laser on probe laser propagation. We found the conditions when the undistorted probe pulse in such a medium, i.e., the EIT effect, is established. Under EIT conditions, the ground state population is trapped in a dark state.
\end{abstract}

The EIT is a quantum interference effect that permits propagation of light through an opaque atomic medium with a significant reduction of absorption. The EIT effect was proposed theoretically in 1989 [1] and experimentally verified in 1991 [2]. Since then, the EIT effect has attracted a tremendous interest over the last years due to its unusual properties and promising potential applications, e.g., laser without inversion [3], slow-light [4], enhancement of Kerr nonlinearity [5, 6], and so on. In addition to applications from the point of view of the atomic system and its optical response, pulse propagation under EIT conditions has also been widely studied, such as: propagation of soliton-like pulses in a three-level system [7], pulse propagation in a prepared energy medium [8], adiabatic propagation of short laser pulses [9], dynamical control of pulse propagation [10-14], etc.

Recently, there has been investigated the influence of pulse areas as well as intensity of pulse propagation under EIT conditions in a three-level $\Lambda$-type atomic medium in terms of the probability amplitudes [15]. It was shown that, when the pulse duration is as short as sub-ps or ps and the pulse areas of the coupling laser are small, the EIT effect does not occur. As the pulse duration become longer and the pulse areas become larger, the EIT is established. If one increases the peak intensities of a coupling laser pulse, the EIT effect is established at shorter pulse duration.

More recently, we have investigated the EIT effect in a five-level cascade system in a steady regime [16]. In this work, using the properties of EIT we study the propagation dynamics of a single pair of short laser pulses in a three-level cascade atomic medium. Unlike in the

\footnotetext{
*E-mail: bangnh@vinhuni.edu.vn
}

case of the three-level $\Lambda$-type atomic medium in Ref. [15], a density matrix method is used here to take into account the population relaxation of excited states which may influence pulse propagation dynamics. The influences of pulse duration, intensity, and pulse area of the coupling laser on probe laser propagation are investigated. Under EIT conditions, the behavior of a population of atomic states is also considered.

We consider a three-level cascade scheme excited by two probe and coupling laser fields as shown in Fig. 1. Here, $|1\rangle$ is the ground state, $|2\rangle$ and $|3\rangle$ are the excited states. A weak probe laser pulse drives the transition $|1\rangle \leftrightarrow|2\rangle$, and an intense coupling laser pulse excites the transition $|2\rangle \leftrightarrow|3\rangle$.

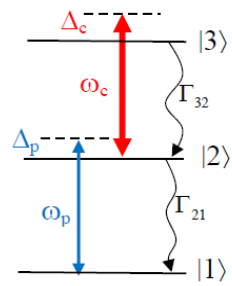

Fig. 1. Scheme of a three-level cascade atomic system.

We assume that both probe and coupling pulses copropagate along the $\mathrm{z}$-axes and the laser fields are described classically as a time and spatially dependent electric field. So, the total electric field vector is written as:

$$
\mathbf{E}=\mathbf{e}_{p} \mathrm{E}_{p}(z, t) e^{-i\left(\omega_{p} t-k_{p} z\right)}+\mathbf{e}_{c} \mathrm{E}_{c}(z, t) e^{-i\left(\omega_{c} t-k_{c} z\right)}+c . c,
$$

where, $\mathcal{E}_{p}(z, t)=\mathcal{E}_{p 0} f_{p}(z, t)$ and $\mathcal{E}_{c}(z, t)=\mathcal{E}_{c 0} f_{c}(z, t)$ are the amplitudes of the electric fields; $f_{p}(z, t)$ and $f_{c}(z, t)$ are the dimensionless pulse envelopes of the probe and coupling laser fields; $k_{p}$ and $k_{c}$ are the wave numbers, $\mathbf{e}_{p}$ and $\mathbf{e}_{c}$ are the polarization vectors, respectively.

The evolution of density matrix elements is represented by [7]:

$$
\dot{\rho}=-\frac{i}{\hbar}[H, \rho]+\Lambda \rho
$$


Where, the total Hamiltonian $H$ is given by:

$$
H=\sum_{i=1}^{3} \hbar \omega_{i}|i\rangle\left\langle i\left|+\Omega_{p} e^{-i\left(\omega_{p} t-k_{p} z\right)}\right| 1\right\rangle\left\langle 2\left|+\Omega_{c} e^{-i\left(\omega_{c} t-k_{c} z\right)}\right| 2\right\rangle\langle 3|+c . c .
$$

Here, $\Omega_{p}=2 d_{12} \mathrm{E}_{p} / \hbar$ and $\Omega_{c}=2 d_{23} \mathrm{E}_{c} / \hbar$ are Rabi frequencies induced by the probe and coupling laser fields, respectively; $d_{12}$ and $d_{23}$ are the dipole matrix elements for the transitions $|1\rangle \leftrightarrow|2\rangle$ and $|2\rangle \leftrightarrow|3\rangle$, respectively; $\Lambda \rho$ presents the relaxation mechanisms of this system.

From Eq. (2), we derive the density matrix equations for the populations and coherences, as follows:

$$
\begin{gathered}
\dot{\rho}_{11}=\Gamma_{21} \rho_{22}+\frac{i}{2} \Omega_{p} \rho_{21}-\frac{i}{2} \Omega_{p}^{*} \rho_{12}, \\
\dot{\rho}_{22}=-\Gamma_{21} \rho_{22}+\Gamma_{32} \rho_{33}+\frac{i}{2} \Omega_{p}^{*} \rho_{12}-\frac{i}{2} \Omega_{p} \rho_{21} \\
+\frac{i}{2} \Omega_{c} \rho_{32}-\frac{i}{2} \Omega_{c}^{*} \rho_{23}, \\
\dot{\rho}_{33}=-\Gamma_{32} \rho_{33}-\frac{i}{2} \Omega_{c} \rho_{32}+\frac{i}{2} \Omega_{c}^{*} \rho_{23}, \\
\dot{\rho}_{12}=-\left(i \Delta_{p}+\gamma_{21}\right) \rho_{12}+\frac{i}{2} \Omega_{p}\left(\rho_{22}-\rho_{11}\right)-\frac{i}{2} \Omega_{c}^{*} \rho_{13}, \\
\dot{\rho}_{23}-\left(i \Delta_{c}+\gamma_{32}\right) \rho_{23}+\frac{i}{2} \Omega_{c}\left(\rho_{33}-\rho_{22}\right)+\frac{i}{2} \Omega_{p}^{*} \rho_{13}, \\
\dot{\rho}_{13}=-\left[i\left(\Delta_{p}+\Delta_{c}\right)+\gamma_{31}\right] \rho_{13}+\frac{i}{2} \Omega_{p} \rho_{23}-\frac{i}{2} \Omega_{c} \rho_{12},
\end{gathered}
$$

with $\rho_{11}+\rho_{22}+\rho_{33}=1$ and $\rho_{j i}=\rho_{i j}^{*}$. Also, $\Delta_{p}=\omega_{p}-\omega_{21}$ and $\Delta_{c}=\omega_{c}-\omega_{32}$ are the probe and coupling frequency detunings, respectively.

Using a slowly varying envelope and rotating-wave approximations, we can write the propagation equations of the probe and coupling fields as:

$$
\begin{aligned}
& \left(\frac{\partial}{\partial z}+\frac{1}{c} \frac{\partial}{\partial t}\right) \Omega_{p}(z, t)=2 i \mu_{p} \rho_{21}(z, t), \\
& \left(\frac{\partial}{\partial z}+\frac{1}{c} \frac{\partial}{\partial t}\right) \Omega_{c}(z, t)=2 i \mu_{c} \rho_{32}(z, t) .
\end{aligned}
$$

Here, $\mu_{m}=\omega_{m} N\left|d_{n 2}\right|^{2} /\left(2 \varepsilon_{0} c \hbar\right)$ is the propagation constant and $n=1$ or 3 , and $N$ is the density of the particle, $\varepsilon_{0}$ is the vacuum permittivity. It is convenient to transform Eqs. (4) and (5) in the local frame where $\xi=z$ and $\tau=t-z / c$, with $\mathrm{c}$ is the speed of light in vacuum. In this frame Eq. (4) will be the same with the substitution $t \rightarrow \tau$ and $z \rightarrow \xi$, while Eq. (5) are rewritten as:

$$
\begin{aligned}
& \frac{\partial}{\partial \xi} \Omega_{p}(\xi, \tau)=2 i \mu_{p} \rho_{21}(\xi, \tau), \\
& \frac{\partial}{\partial \xi} \Omega_{c}(\xi, \tau)=2 i \mu_{c} \rho_{32}(\xi, \tau) .
\end{aligned}
$$

In order to investigate the propagation dynamics of a pair of probe and coupling pulses in a three-level cascade atomic medium, we numerically solved the coupled Bloch-Maxwell Eqs. (4) and (6) for atom and field. The propagation length is represented in units of $\mu_{p} \xi$ which is the so-called optical depth [18]. The temporal profile of the fields at the entrance to the medium is a Gaussian function, i.e.,

$$
f(\xi=0, \tau)=e^{-\pi\left(\tau / \tau_{0}\right)^{2}},
$$

with $\tau_{0}$ is the pulse temporal duration of a laser pulse, which is assumed to be identical for both probe and coupling lasers.
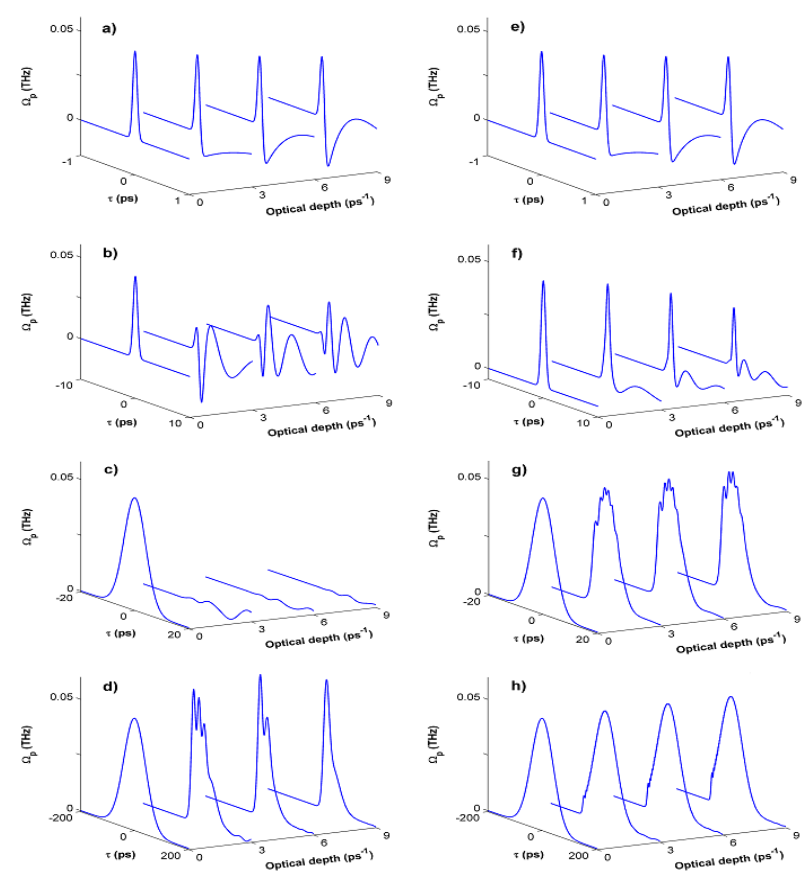

Fig. 2. Temporal variation of the probe laser field $\Omega_{\mathrm{p}}(\xi, \tau)$ at different optical depths $\mu_{\mathrm{p}} \xi=0,3,6$ and $9 \mathrm{ps}^{-1}$ when we irradiate a single pair of probe and coupling laser pulses with the durations of $\tau_{0}=0.1 \mathrm{ps}[(\mathrm{a})$ and (e)], 1 ps [(b) and (f)], $10 \mathrm{ps}[(\mathrm{c})$ and (g)], $100 \mathrm{ps}[(\mathrm{d})$ and (h) ]. The coupling Rabi frequency $\Omega_{\mathrm{c} 0}=1 \mathrm{THz}$ for graphs [(a)-(d)], and $\Omega_{\mathrm{c} 0}=10$ $\mathrm{THz}$ for graphs [(e)-(h)]. The other parameters are $\Omega_{\mathrm{c} 0}=0.05 \mathrm{THz}, \Delta_{\mathrm{p}}=$ $\Delta_{\mathrm{c}}=0, \Gamma_{21}=6 \mathrm{MHz}, \Gamma_{32}=1 \mathrm{MHz}$ for all graphs.

The influences of pulse duration and intensity of the coupling laser on the propagation of a probe laser have been displayed in Fig. 2. In Figure 2, we plot the temporal shape of the probe laser pulse $\Omega_{p}(\xi, \tau)$ at different optical 
depths $\mu_{p} \xi=0,3,6$ and 9 ps $^{-1}$. From Figs. 2(a)-(c) and Figs. 2(e)-(f), we see that for small and moderate coupling pulse areas $\left(\Omega_{c 0} \tau_{0} \leq 10\right)$ the probe laser pulse is significantly distorted during propagation, and each pulse breaks up into several sub-pulses with positive and negative amplitudes [16, 19]. The number of oscillations at the trailing edge of the pulse increases as the propagation distance increases. The physical reason for these oscillations at the trailing edge of the pulse is explained as follows. When the pulse duration (sub-ps or ps) is shorter than the lifetime of the excited state $|2\rangle$ and therefore the pulse area is small, the EIT effect has not yet appeared. In this case, we simply observe the oscillations at the trailing edge of the probe pulse [16, 19], as shown in Fig. 2(a) and (b). When the pulse duration increases to $100 \mathrm{ps}$ and therefore the pulse area becomes larger $\left(\Omega_{\mathrm{c} 0} \tau_{0}\right.$ $>10$ ), the EIT begins to appear, i.e., the number of modulations at the trailing edge of the pulse and the pulse distortion decreases significantly during the propagation, as shown in Fig. 2(d). If we increase the peak intensity of the coupling laser pulse to 10 times, the EIT effect occurs at shorter pulse durations [see Figs. 2(g), (d)]. Specially, when the pulse duration $\tau_{0}=100 \mathrm{ps}$ and the coupling laser intensity $\Omega_{\mathrm{c} 0}=10 \mathrm{THz}$, the probe laser pulse propagates in the medium almost undistorted and is not oscillated at the trailing edge of the pulse, as illustrated in Fig. 2(h).
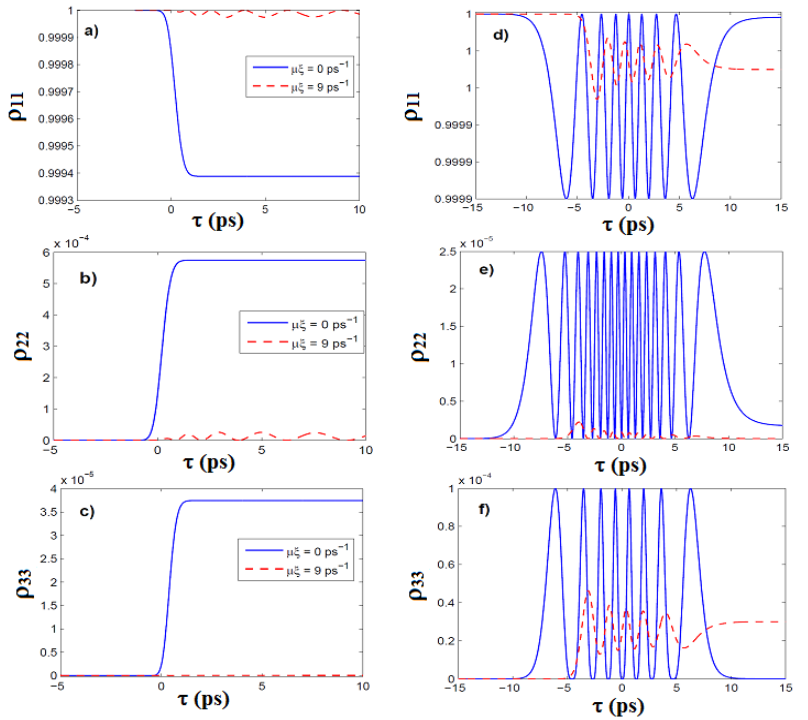

Fig. 3. Temporal variation of populations at the start $\left(\mu_{p} \xi=0\right)$ and end $\left(\mu_{\mathrm{p}} \xi=9 \mathrm{ps}^{-1}\right)$ of the medium. The employed parameters in the left and right column are similar to those in Fig. 2(b) and 2(g), respectively.

Other parameters are the same as those in Fig. 2.

In Figure 3, we examine the behavior of atoms in a steady dark state by considering the variation of states population versus the time at two different optical depths $\mu_{\mathrm{p}} \xi=0$ and $9 \mathrm{ps}^{-1}$ for two cases corresponding to Fig. 2(b) without EIT and Fig. 2(g) with EIT. It can be realized from Fig. 3 that for the case without EIT, at $\mu_{\mathrm{p}} \xi=0$ the atoms exhibit strong Rabi flopping in their populations, while at the end of the medium, $\mu_{\mathrm{p}} \xi=9 \mathrm{ps}^{-1}$, the excited state populations are much less pronounced [9] and they oscillate unstably. However, when EIT is established, the atomic population is trapped in a steady dark state which is equivalent to $|1\rangle$. Their populations oscillate stably in time at the start and end of the medium, as seen in the right column of Fig. 3.

We have investigated the dynamics of pulse propagation in a three-level cascade atomic medium by means of density matrix formalism. Considering the influences of pulse duration, intensity, and pulse area of the coupling laser, we found the conditions when the atomic medium becomes transparent for the probe laser pulse. Thus, the optical pulses propagating in an optical thick medium are undistorted, i.e., the soliton-like pulses are observed. When the EIT is established, the ground state population is trapped in the steady dark state.

\section{References}

[1] A. Imamoglu, S.E. Harris, Opt. Lett. 14, 1344 (1989).

[2] K.J. Boller, A. Imamoglu, S.E. Harris, Phys. Rev. Lett. 66, 2593 (1991).

[3] A.S. Zibrov, M.D. Lukin, D.E. Nikonov, L. Hollberg, M.O. Scully, V.L. Velichansky, H.G. Robinson, Phys. Rev. Lett. 75, 1499 (1995).

[4] L.V. Hau, S. E. Harris, Z. Dutton, C.H. Bejroozi, Nature 397, 594 (1999).

[5] L.V. Doai, D.X. Khoa, N.H. Bang, Phys. Scr. 90, 045502 (2015).

[6] D.X. Khoa, L.V. Doai, D.H. Son, N.H. Bang, J. Opt. Soc. Am. B, 31, N6, 1330 (2014).

[7] F.T. Hioe, R. Grobe, Phys. Rev. Lett. 73, 2559 (1994).

[8] S.E. Harris and Z.F. Luo, Phys. Rev. A. 52, R928 (1995).

[9] V.G. Arkhipkin, I.V. Timofeev, Quantum Electronics 30, 180 (2000).

[10] M. Kiffner, T.N. Dey, Phys. Rev. A. 79, 023829 (2009).

[11] R. Yu, J. Li, P. Huang, A. Zheng, X. Yang, Phys. Lett. A. 373, 2992 (2009)

[12] E. Paspalakis, N.J. Kylstra, P.L. Knight, Phys. Rev. A 65, 053808 (2002).

[13] E. Ignesti, R. Buffa, L. Fini, E. Sali, M. V. Tognetti, S. Cavalieri, Opt. Commun, 285, 1185 (2012).

[14] P.R. Bermanand, C.H. Raymond Ooi, Phys. Rev. A 86, 053812 (2012).

[15] G. Buica, T. Nakajima, Opt. Commun. 332, 59 (2014).

[16] L.V. Doai, P.V. Trong, D.X. Khoa, Ng. H. Bang, Optik, 125, 3666 (2014).

[17] J.H. Eberly, Quantum Semiclass. Opt. 7, 373 (1995).

[18] M.D. Crisp, Phys. Rev. A 1, 1604 (1970). 